\title{
NÚMERO DE ACCIDENTES CAUSADOS POR ANIMALES VENENOSOS ENTRE 2010 Y 2014 EN BRASIL
}

\section{ARTÍCULO ORIGINAL}

SOEIRO, Selthon Macello Capiberibe ${ }^{1}$, FACCO, Lucas², FECURY, Amanda Alves ${ }^{3}$, ARAÚJO, Maria Helena Mendonça de ${ }^{4}$, OLIVEIRA, Euzébio de ${ }^{5}$, DENDASCK, Carla $V_{\text {Viana }}^{6}$, SOUZA, Keulle Oliveira da ${ }^{7}$, DIAS, Claudio Alberto Gellis de Mattos ${ }^{8}$

SOEIRO, Selthon Macello Capiberibe. Et al. Número de accidentes causados por animales venenosos entre 2010 y 2014 en Brasil. Revista Científica Multidisciplinar Núcleo do Conhecimento. Año 05, Ed. 12, Vol. 01, págs. 05-17. Diciembre de 2020. ISSN: 2448-0959, Enlace de acceso: https://www.nucleodoconhecimento.com.br/salud/numero-de-accidentes, DOI: 10.32749/nucleodoconhecimento.com.br/salud/numero-de-accidentes

\section{RESUMEN}

Los animales venenosos son aquellos que tienen un mecanismo para inyectar veneno. Diferentes venenos o venenos pueden causar dolor, ruptura de los vasos sanguíneos, quemaduras, ampollas, edemas, lesiones necróticas, hemorragia, vómitos sanguíneos, presencia de sangre en la orina, sangrado en la nariz, sudoración intensa, presión arterial baja, hipotermia y problemas neurológicos. El objetivo de este artículo es mostrar el número de accidentes causados por animales

\footnotetext{
${ }^{1}$ Graduación en curso en Ciencias de la Computación.

${ }^{2}$ Estudiante del Curso de Medicina de la Universidad Federal de Amapá (UNIFAP).

${ }^{3}$ Biomédica, Doctora en Enfermedades Tropicales, Profesora e investigadora del Curso Médico de la Universidad Federal de Amapá (UNIFAP).

${ }^{4}$ Médico, Profesor e investigador del Curso Médico de la Universidad Federal de Amapá (UNIFAP).

${ }^{5}$ Biólogo, Doctor en Enfermedades tópicas, Profesor e investigador del Curso de Educación Física de la Universidad Federal de Pará (UFPA).

${ }^{6}$ Teólogo, Doctor en Psicoanálisis, investigador del Centro de Investigación y Estudios Avanzados - CEPA.

${ }^{7}$ Sociólogo, Máster en Estudios Antrópicos en la Amazonía, Miembro del Grupo de Investigación "Laboratorio de Educación, Medio Ambiente y Salud" (LEMAS/UFPA).

${ }^{8}$ Biólogo, Doctor en Teoría e Investigación del Comportamiento, Profesor e investigador del Programa de Posgrado en Educación Profesional y Tecnológica (PROFEPT), Instituto Federal de Amapá (IFAP).
}

RC: 67469

Disponible: https://www.nucleodoconhecimento.com.br/salud/numero-de-accidentes 
venenosos entre 2010 y 2014 en Brasil. Considerados como causas desatendidas de enfermedades, los accidentes causados por animales venenosos requieren una mayor atención de salud pública. Arañas, escorpiones y serpientes son los principales causadores de estos accidentes. Entre los niños, los niños son los más afectados, y la falta de supervisión en las actividades diarias es una de las principales causas de este problema. La producción de suero para el tratamiento de accidentes de odidisa en Brasil se acentúa, y la cantidad producida es capaz de cubrir el número total de casos en el país. Sin embargo, existe un problema con respecto a la distribución de este suero, ya que las zonas de población indígena y regiones de localidades remotas no tienen una disponibilidad adecuada, lo que permite consecuencias negativas para los individuos en cuestión, aumentando la posibilidad de muertes por este tipo de accidentes.

Palabras clave: Epidemiología, veneno, veneno.

\section{INTRODUCCIÓN}

Los animales venenosos son aquellos que tienen un mecanismo para inyectar veneno, que puede ser a picadores, a picadores o dientes (Pinho y Pardal, 2015). Algunas especies de abejas, serpientes, avispas, marimbondos, arañas, escorpiones y orugas pueden considerarse animales venenosos (BREDT y LITCHTENEKER, 2014; NEIVA, et al., 2019).

Diferentes venenos o venenos pueden causar dolor, ruptura de los vasos sanguíneos, quemaduras, ampollas, edemas, lesiones necróticas, hemorragia, vómitos sanguíneos, presencia de sangre en la orina, sangrado en la nariz, sudoración grave, presión arterial baja, hipotermia y problemas neurológicos (LIMA et al., 2017; NEIVA, et al., 2019).

El tratamiento de accidentes con animales venenosos se realiza, en la mayoría de los casos, mediante un suero antídoto que contiene anticuerpos específicos para

RC: 67469

Disponible: https://www.nucleodoconhecimento.com.br/salud/numero-de-accidentes 
combatir las toxinas de cada animal, y esto debe aplicarse lo antes posible para evitar el agravamiento del caso (SOUZA y MACHADO, 2017).

Para evitar accidentes de serpientes, se deben usar guantes y botas al limpiar arbustos, escombros, hojas secas, basura y otros, ya que estos animales prefieren lugares calientes y húmedos. No se debe poner las manos en agujeros, se debe evitar la proliferación de ratas, ya que se trata de alimento de serpiente y, además, es necesario evitar la acumulación de arbustos, madera, ladrillos y otros materiales que pueden convertirse en un refugio para los animales que sirven como alimento para las serpientes (BRASIL, 2001).

Para evitar accidentes con arácnidos, páramo, jardines y patios traseros deben mantenerse libres de escombros, arbustos, hojas secas y materiales de construcción, evitando así que encuentren refugio. Además, uno debe: evitar las plantas con follaje denso cerca de las paredes; agitar la ropa y los zapatos antes de usar; usar guantes y zapatos al manipular y caminar en lugares que puedan contener tales animales; sellar aberturas donde los arácnidos pueden cruzar (principalmente por la noche, debido a sus hábitos); poner pantallas en drenajes, sumideros y tanques; prevenir la proliferación de insectos que son el alimento de los arácnidos (una de las medidas es mantener los residuos domésticos en el interior para evitar cucarachas y moscas); quitar las camas de las paredes y no dejar que los mosqueteros y sábanas toquen el suelo; evitar matar depredadores arácnidos (ranas, aves, lagartos, pollos, gansos, monos y otros) (BRASIL, 2001).

El número de casos en Brasil entre 1986 y 2016 fue de 2.183.989. El número de casos por región en Brasil, entre 1986 y 2016, es: Región Norte: 233.586; Región Noreste: 624.615; Región Sureste: 758.238; Región Sur: 427.442; Región del Medio Oeste: 140.108. El número de casos en Amapá entre 1986 y 2016 en Amapá 8463 (BRASIL, 2017).

RC: 67469

Disponible: https://www.nucleodoconhecimento.com.br/salud/numero-de-accidentes 


\section{OBJETIVOS}

Mostrar el número de accidentes causados por animales venenosos entre 2010 y 2014 en Brasil.

\section{MÉTODO}

La investigación se realizó en la base de datos DATASUS (http://datasus.saude.gov.br/). Los datos nacionales se recolectaron de acuerdo con los siguientes pasos: A) Accedimos al enlace datasus.saude.gov.br, movimos la flecha con el mouse a la pestaña "Acceso a la información", y luego pasamos a la pestaña "Información de salud (TABNET) ", Se hizo clic en“ Epidemiología y morbilidad ". En la página siguiente, haga clic en "Enfermedades y Trastornos Notificados - Desde 2007 en adelante (SINAN)". En la página siguiente seleccionamos "Accidentes por animales venenosos. En el cuadro "Ámbito geográfico", la opción era "Brasil por región, estado y municipio". A partir de entonces se siguieron los pasos: A) En la línea se seleccionó "Año de accidente"; en la columna "No activo", y en el contenido "Notificaciones". Todos los datos recopilados en este y los siguientes pasos son entre 2010 y 2014. B) En la línea, se seleccionó "Año del accidente"; en la columna "Mes del accidente", y en el contenido "Notificaciones". C) En la línea, se seleccionó "Año del accidente"; en la columna "Región de notificación", y en el contenido "Notificaciones". D) En la línea, se seleccionó "Año del accidente"; en la columna "Grupo de edad" y en el contenido "Notificaciones". E) En la línea, se seleccionó "Año del accidente"; en la columna "Raza", y en el contenido "Notificaciones". F) En la línea se seleccionó "Año del accidente"; en la columna "Sexo", y en el contenido "Notificaciones". G) En la línea, se seleccionó "Año del accidente"; en la columna "Mujer embarazada", y en el contenido "Notificaciones". H) En la línea, se seleccionó "Año del accidente"; en la columna "Corte de tiempo / servicio", y en el contenido "Notificaciones". I) En la línea se seleccionó "Año de accidente"; en la columna "Tipo de Accidente", y en el contenido "Notificaciones". J) En la línea, se seleccionó "Año del accidente"; en la

RC: 67469

Disponible: https://www.nucleodoconhecimento.com.br/salud/numero-de-accidentes 
columna "Tarifa. Final ", y en el contenido" Notificaciones ". K) En la línea, se seleccionó "Año del accidente"; en la columna "Evolución del caso" y en el contenido "Notificaciones"

Los datos se compilaron dentro de la aplicación Excel, un componente de la suite de Microsoft Corporation Office. La investigación bibliográfica se llevó a cabo en artículos científicos, utilizando computadoras del laboratorio de computación del Instituto Federal de Educación, Ciencia y Tecnología de Amapá, Campus Macapá, ubicado en: Rodovia BR 210 KM 3, s/n - Bairro Brasil Novo. Código postal: 68.909398, Macapá, Amapá, Brasil.

\section{RESULTADOS}

La Figura 1 muestra el número de accidentes causados por animales venenosos entre 2010 y 2014 , lo que indica un aumento cada año.

La Figura 1 muestra el número de casos de accidentes causados por animales venenosos en Brasil entre 2010 y 2014.

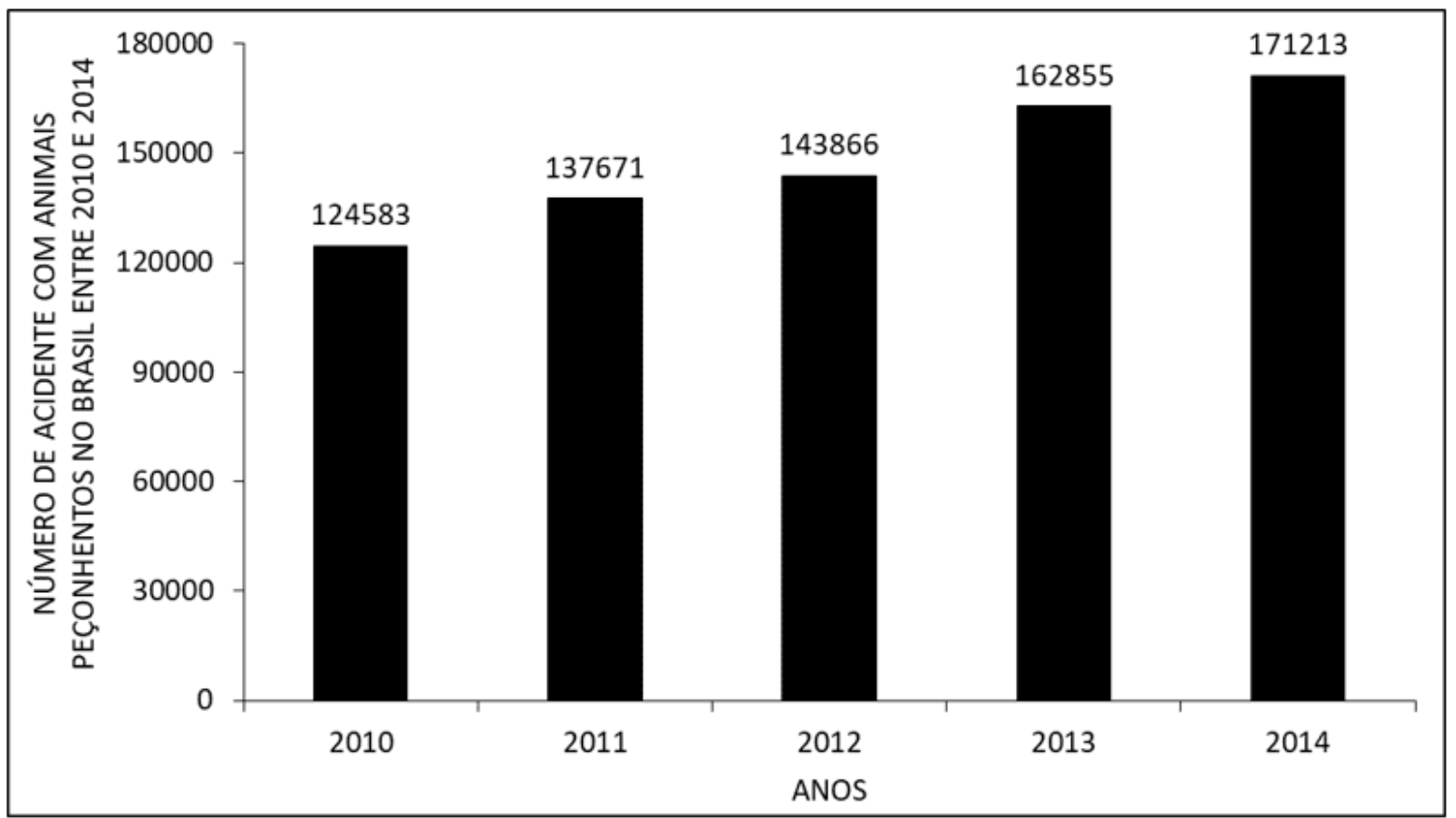

RC: 67469

Disponible: https://www.nucleodoconhecimento.com.br/salud/numero-de-accidentes 
La Figura 2 muestra el número de accidentes causados por animales venenosos por mes del año entre 2010 y 2014. El mayor número se produce en enero, mostrando una caída en febrero, aumentando de nuevo en marzo y de nuevo disminuyendo hasta julio. Este es el mes con el menor número de casos, con un aumento en los meses siguientes.

La Figura 2 muestra el número de casos de accidentes causados por animales venenosos en Brasil entre 2010 y 2014, durante meses del año.

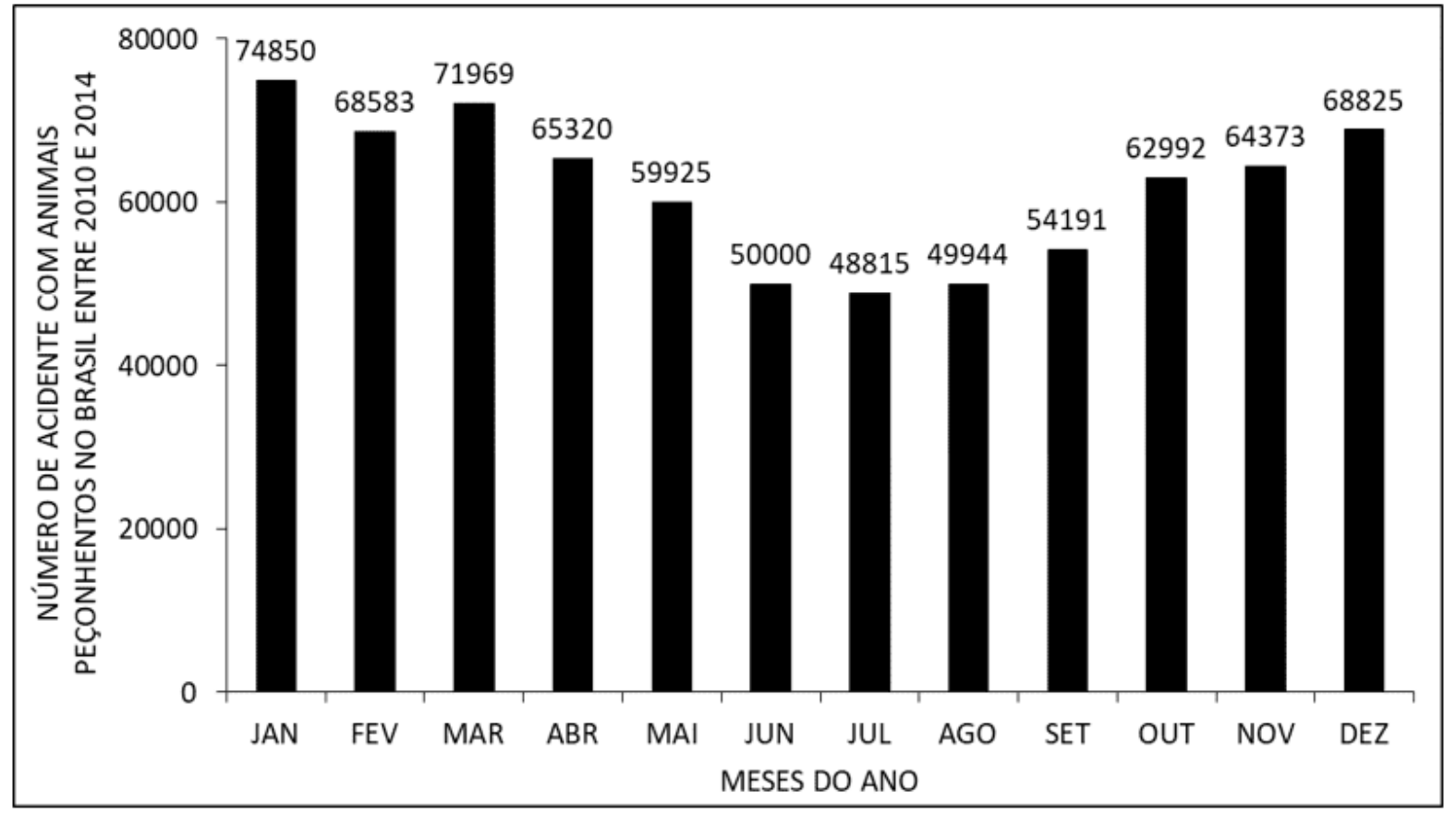

La Figura 3 muestra el número de denuncias de accidentes causados por animales venenosos en Brasil entre 2010 y 2014, por regiones del país. El mayor número de casos ocurre en la región sureste seguido, respectivamente, por las regiones noreste, sur, norte y medio oeste.

RC: 67469

Disponible: https://www.nucleodoconhecimento.com.br/salud/numero-de-accidentes 
La Figura 3 muestra el número de casos de accidentes causados por animales venenosos en Brasil entre 2010 y 2014, por regiones del país.

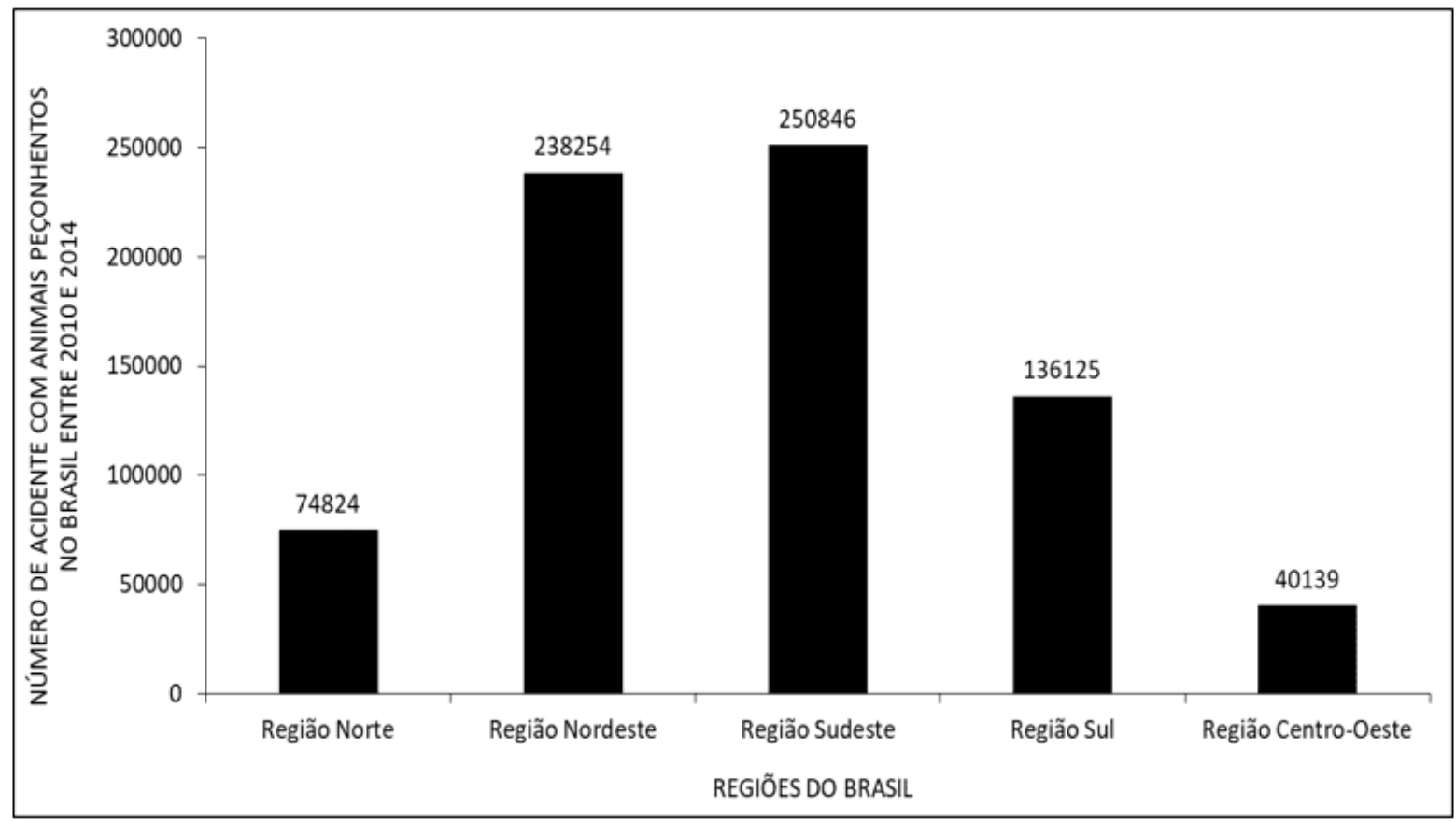

La Figura 4 muestra el número de denuncias de accidentes causados por animales venenosos en Brasil entre 2010 y 2014, por sexo. El mayor número de casos les sucede a los hombres.

RC: 67469

Disponible: https://www.nucleodoconhecimento.com.br/salud/numero-de-accidentes 
La Figura 4 muestra el número de casos de accidentes causados por animales venenosos en Brasil entre 2010 y 2014, por sexo.

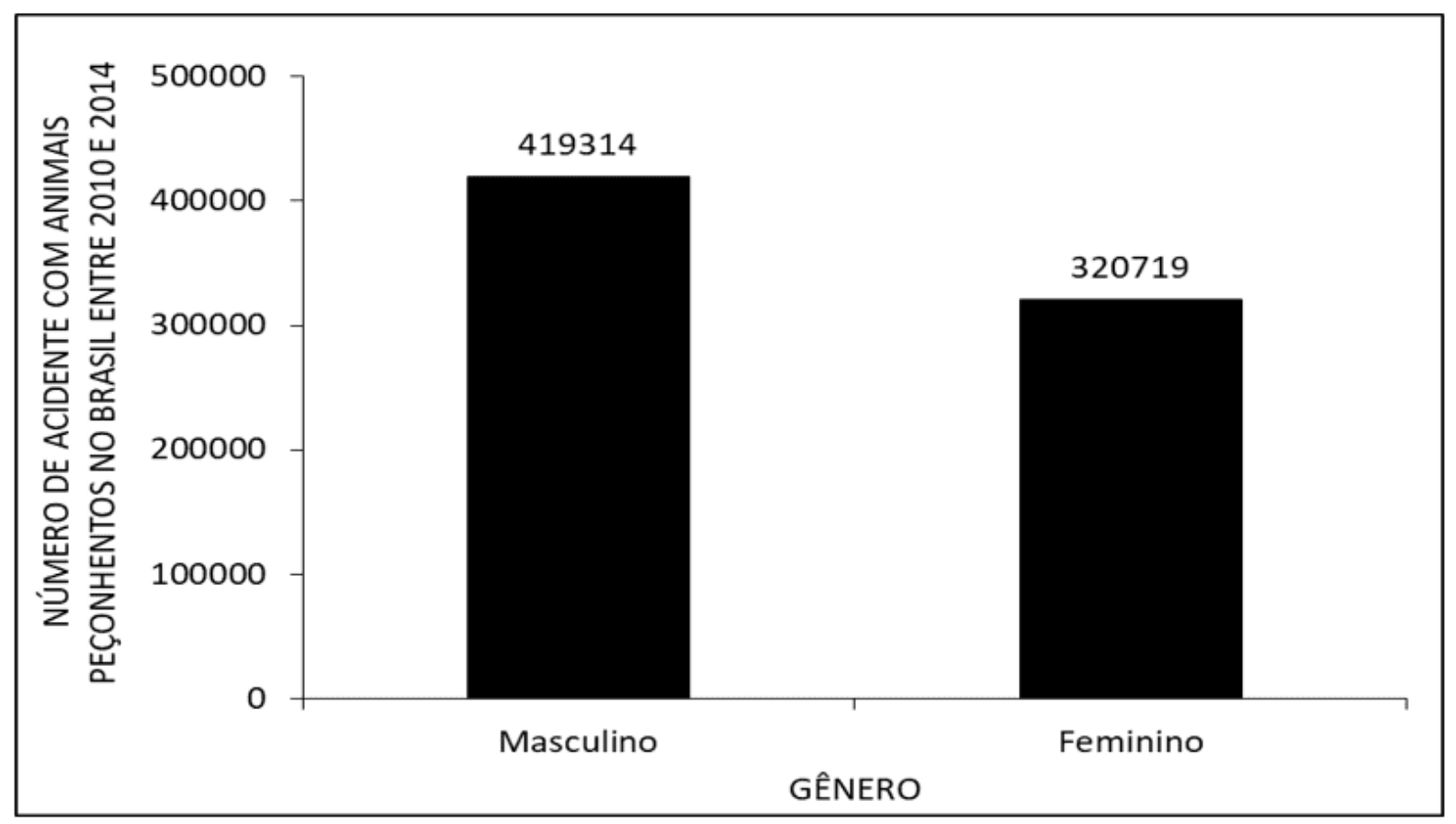

La Figura 5 muestra el número de denuncias de accidentes causados por animales venenosos en Brasil entre 2010 y 2014, por grupo de edad. El mayor número de casos, según los datos, ocurre con personas de entre 20 y 39 años. El segundo mayor número de casos aparece en el grupo entre 40 y 59 años.

RC: 67469

Disponible: https://www.nucleodoconhecimento.com.br/salud/numero-de-accidentes 
La Figura 5 muestra el número de casos de accidentes causados por animales venenosos en Brasil entre 2010 y 2014, por grupo de edad.

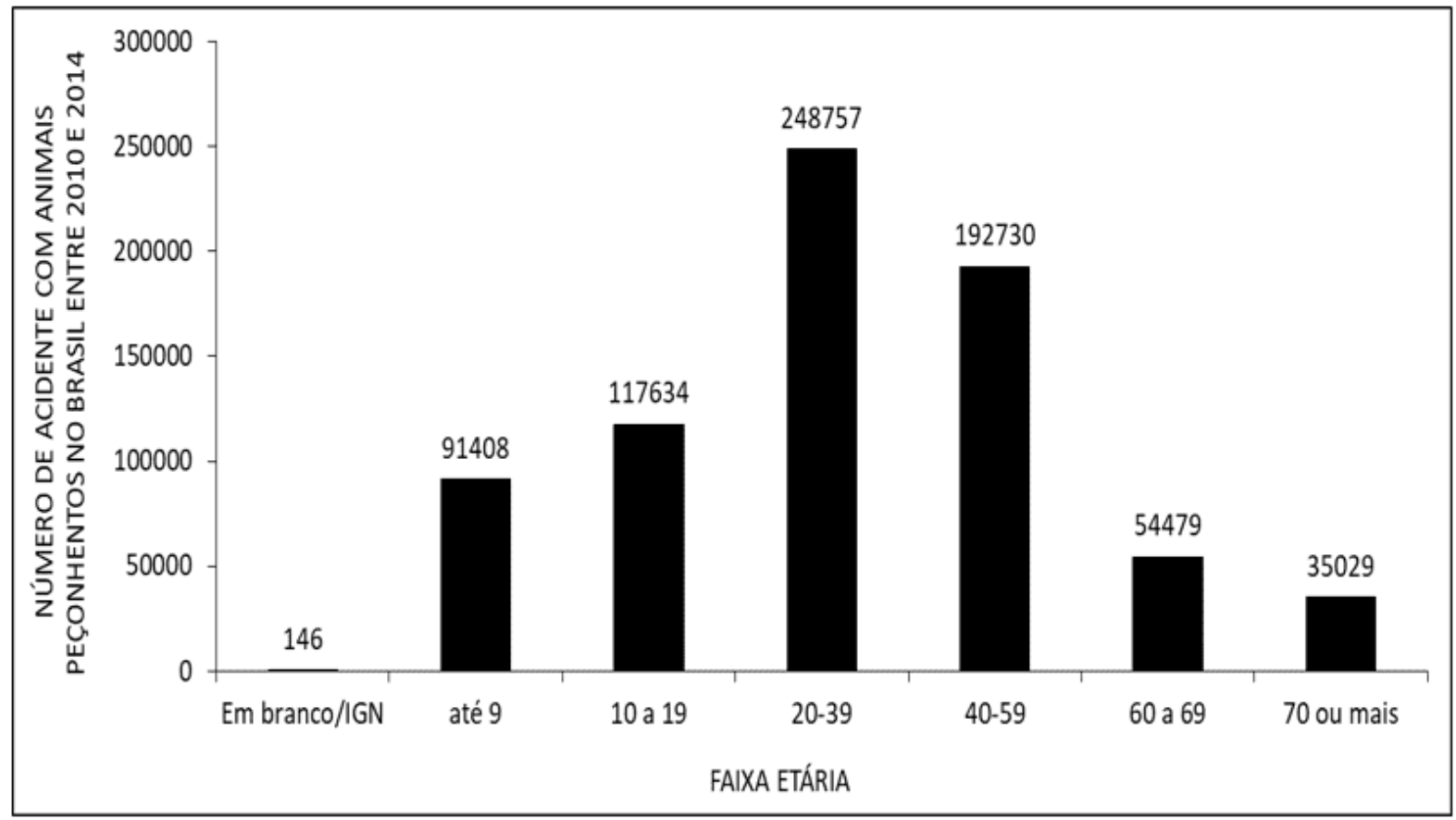

La Figura 6 muestra el número de denuncias de accidentes causados por animales venenosos en Brasil entre 2010 y 2014, por etnia. El mayor número de casos ocurre con personas de etnia marrón, seguidos por personas declaradas blancas y negras, respectivamente.

RC: 67469

Disponible: https://www.nucleodoconhecimento.com.br/salud/numero-de-accidentes 
La Figura 6 muestra el número de casos de accidentes causados por animales venenosos en Brasil entre 2010 y 2014, por etnia.

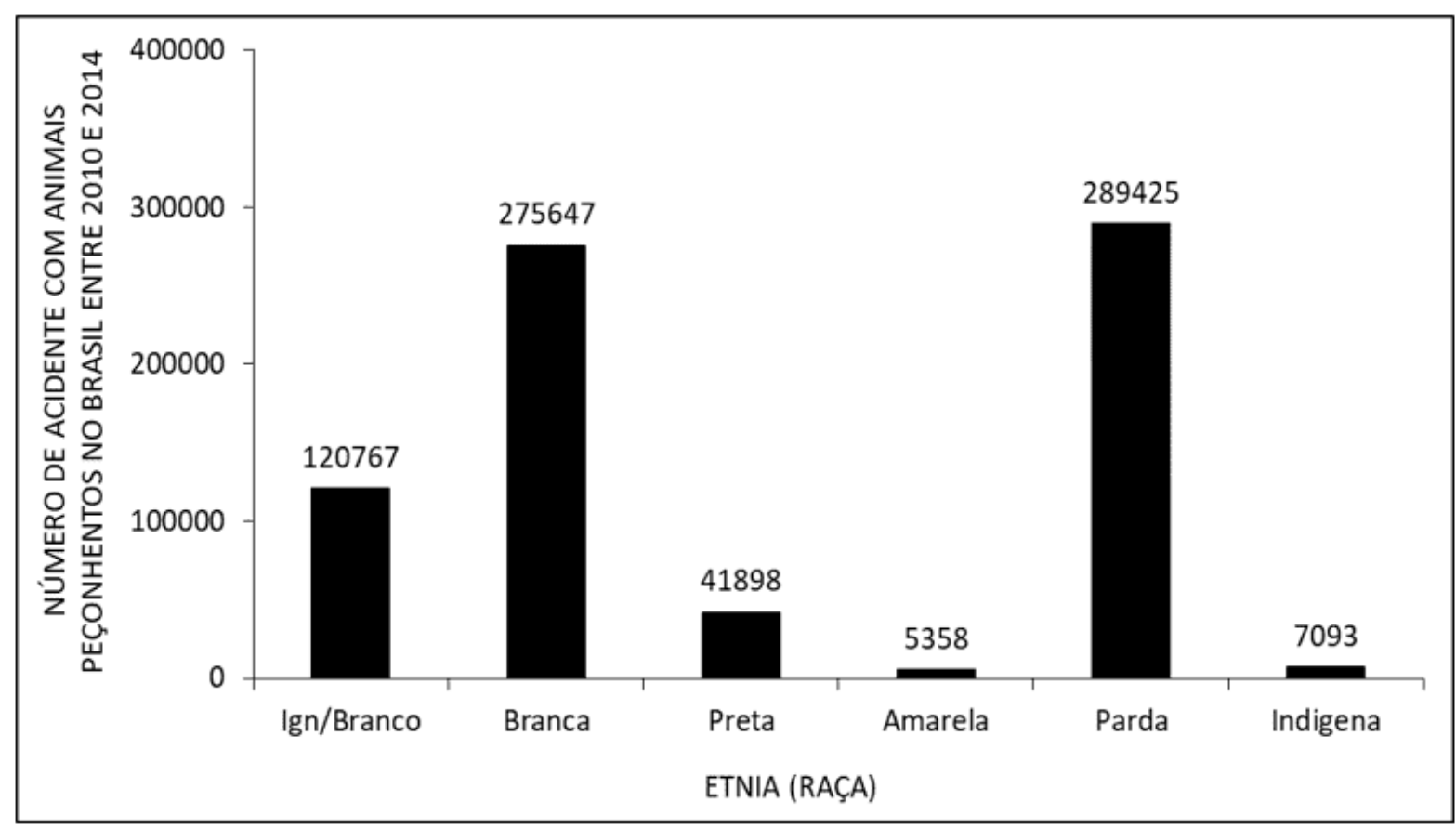

La Figura 7 muestra el número de denuncias de accidentes causados por animales venenosos en Brasil entre 2010 y 2014, por tipo de accidente. El mayor número de casos ocurrieron en accidentes con escorpiones.

RC: 67469

Disponible: https://www.nucleodoconhecimento.com.br/salud/numero-de-accidentes 
La Figura 7 muestra el número de casos de accidentes causados por animales venenosos en Brasil entre 2010 y 2014, por tipo de accidente.

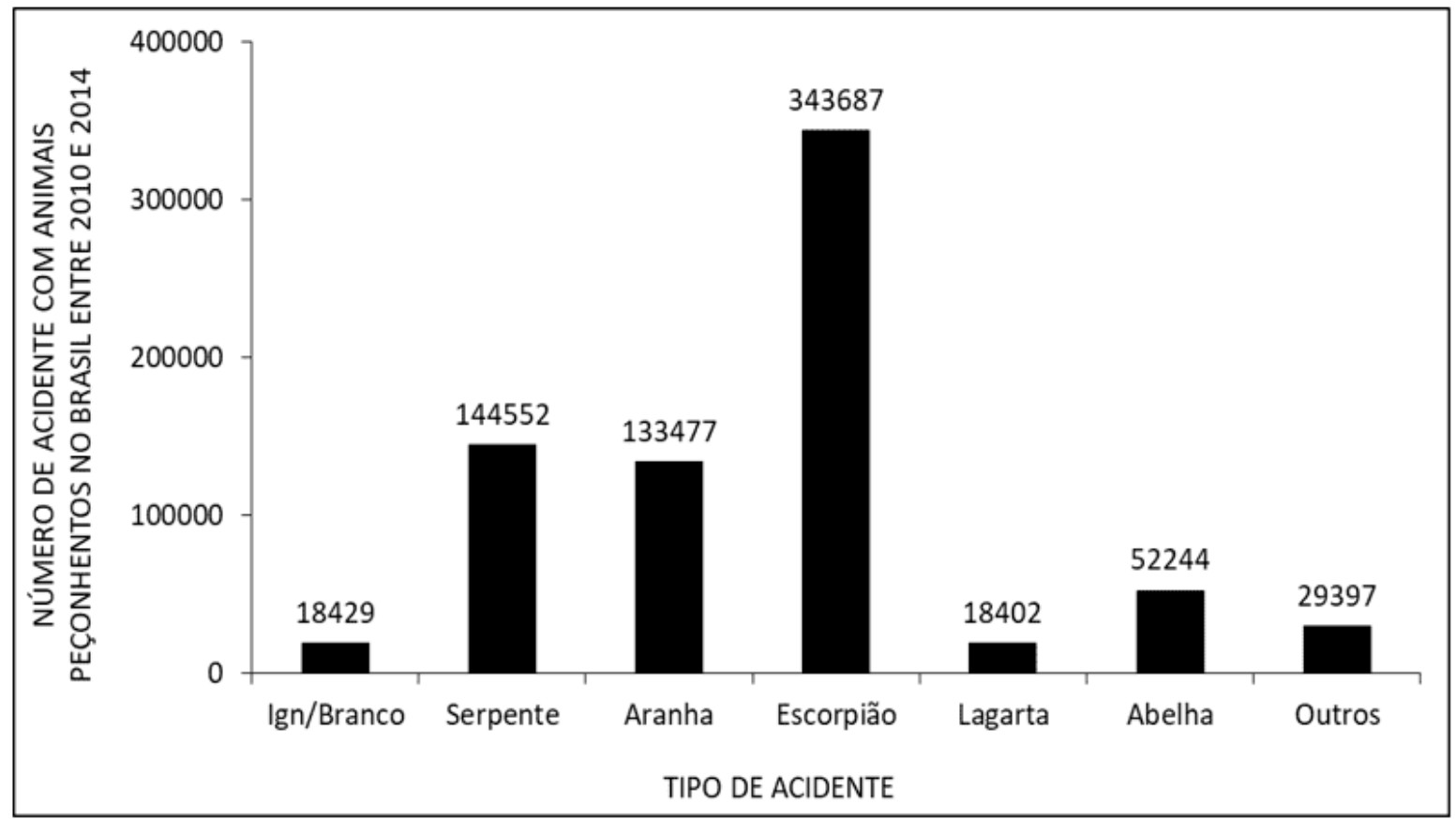

La Figura 8 muestra el número de informes de accidentes causados por animales venenosos en Brasil entre 2010 y 2014, según las etapas del embarazo. El mayor número de casos ocurre con mujeres embarazadas en el segundo trimestre, seguido de mujeres embarazadas en el primer y tercer trimestre, respectivamente.

RC: 67469

Disponible: https://www.nucleodoconhecimento.com.br/salud/numero-de-accidentes 
La Figura 8 muestra el número de casos de accidentes causados por animales venenosos en Brasil entre 2010 y 2014, según las etapas del embarazo.

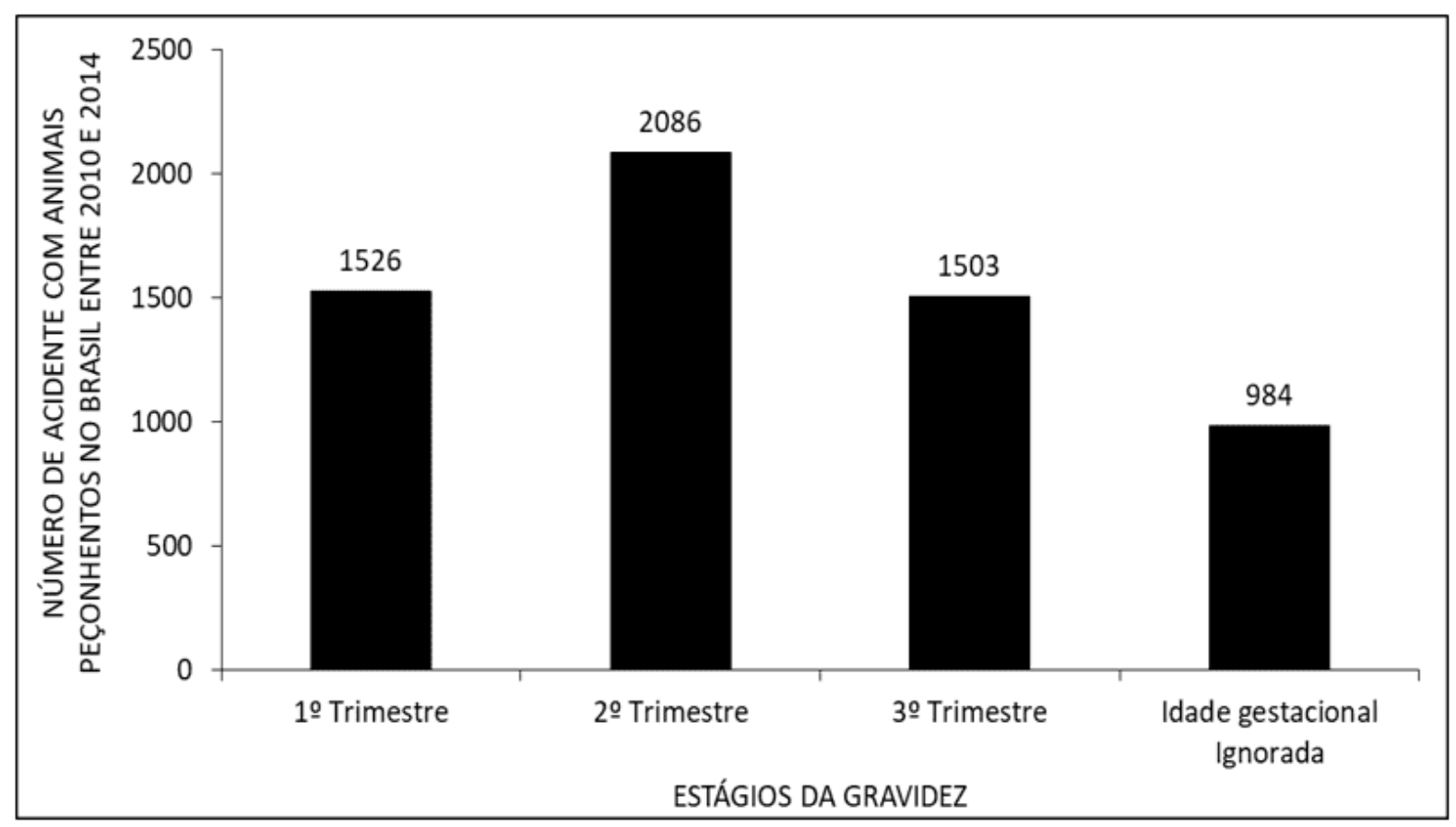

La Figura 9 muestra el número de informes de accidentes causados por animales venenosos en Brasil entre 2010 y 2014, según el tiempo en horas entre la picadura y el servicio. Los datos muestran que el mayor número de personas es atendido entre 0 y 3 horas después del accidente.

RC: 67469

Disponible: https://www.nucleodoconhecimento.com.br/salud/numero-de-accidentes 
La Figura 9 muestra el número de casos de accidentes causados por animales venenosos en Brasil entre 2010 y 2014, según el tiempo en horas entre la picadura y el cuidado.

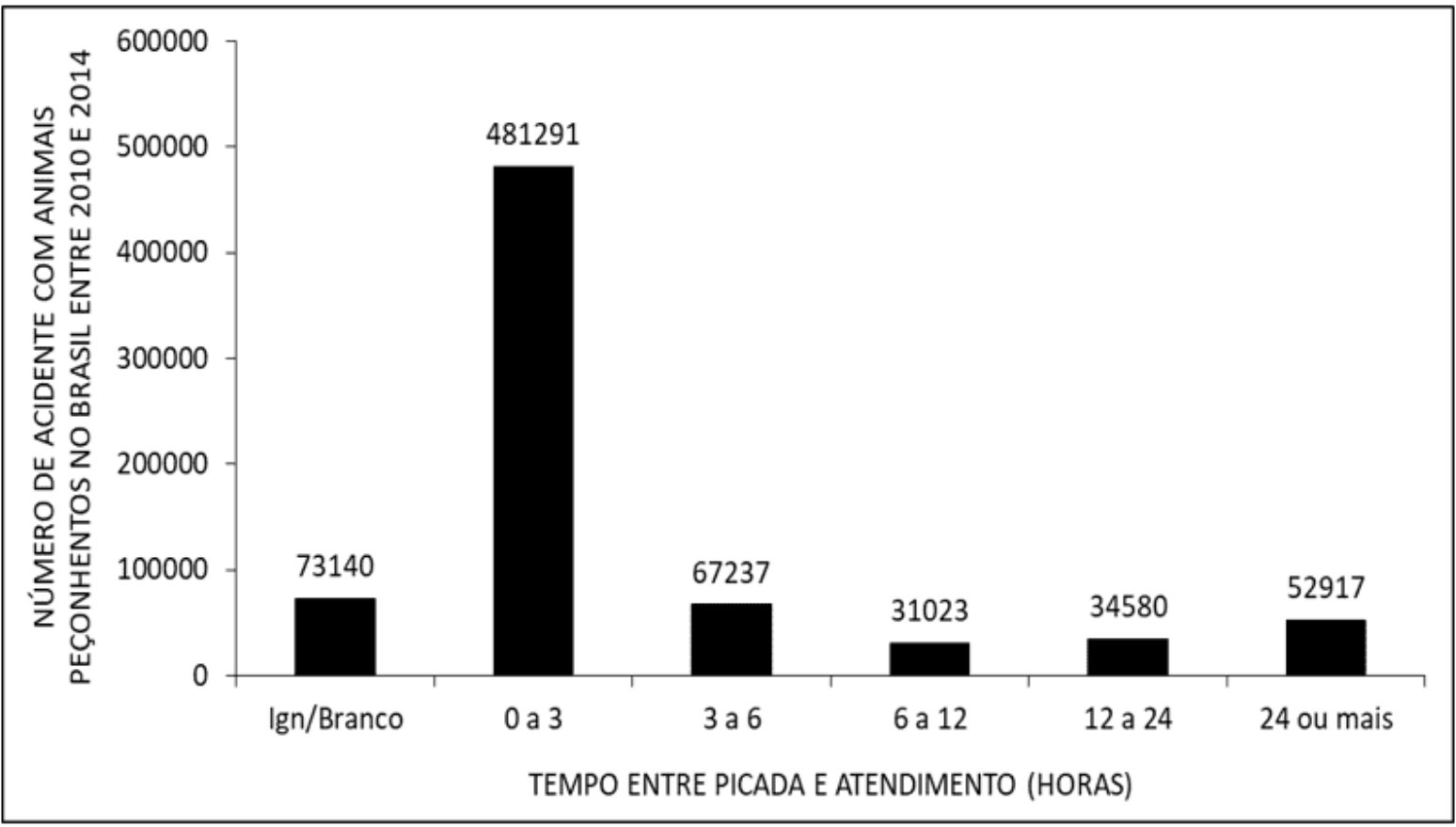

La Figura 10 muestra el número de informes de accidentes causados por animales venenosos en Brasil entre 2010 y 2014, por clasificación final del accidente. El mayor número de casos tuvo como clasificación final el accidente de grado leve.

RC: 67469

Disponible: https://www.nucleodoconhecimento.com.br/salud/numero-de-accidentes 
La Figura 10 muestra el número de casos de accidentes causados por animales venenosos en Brasil entre 2010 y 2014, por clasificación final del accidente.

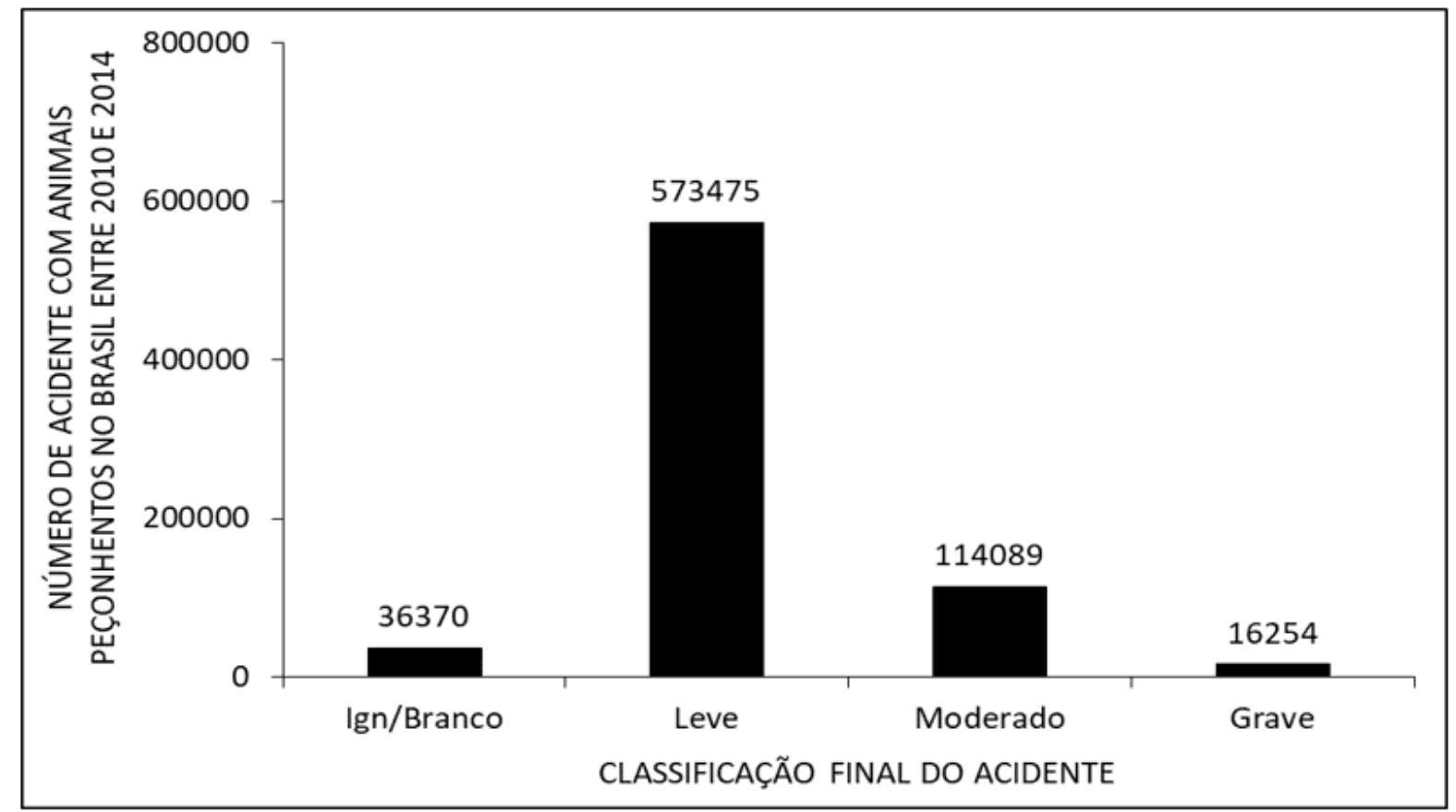

La Figura 11 muestra el número de informes de accidentes causados por animales venenosos en Brasil entre 2010 y 2014, por la evolución del caso. El mayor número de casos se curó, con una cantidad mínima de muerte.

RC: 67469

Disponible: https://www.nucleodoconhecimento.com.br/salud/numero-de-accidentes 
La Figura 11 muestra el número de casos de accidentes causados por animales venenosos en Brasil entre 2010 y 2014, debido a la evolución del caso.

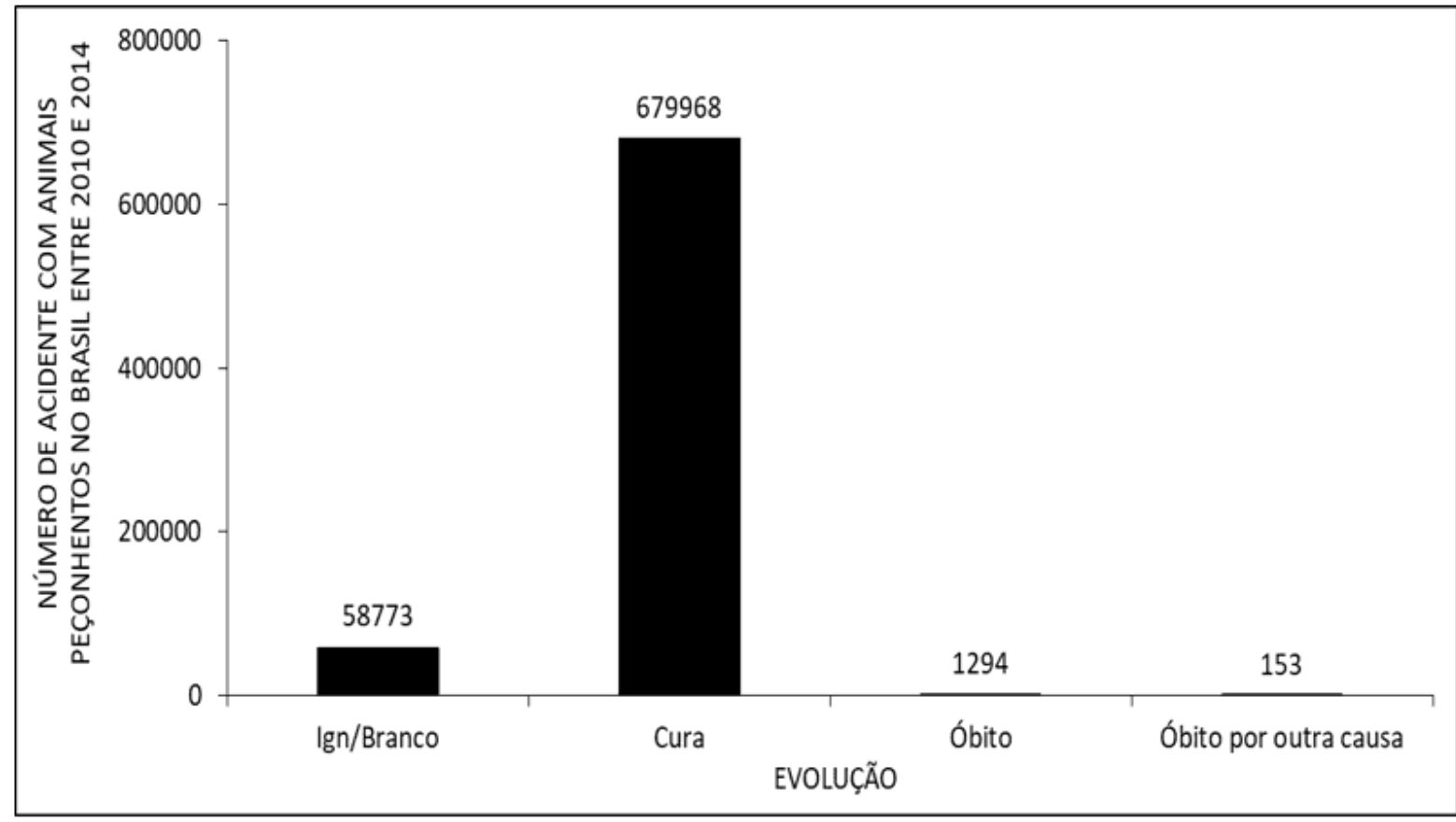

\section{DISCUSIÓN}

Los accidentes causados por animales venenosos se consideran causas desatendidas de enfermedades, y su aparición es mayor en las zonas rurales y en los países cuya pobreza está muy extendida. Capaces de generar una serie de pérdidas, tales accidentes son capaces de causar consecuencias a nivel orgánico y socioeconómico, ya que justifican la necesidad de inversiones en métodos profilácticos y sueros para el tratamiento de tales implicaciones (SALOMÃO et al., 2018). Considerados como una emergencia clínica (es decir, en la que hay una necesidad de atención inmediata al paciente), son causados por una serie de animales venenosos, siendo escorpiones, arañas y serpientes. En 2013, en Brasil, hubo 158.002 casos de accidentes con animaciones venenosas, la mayoría (alrededor del 78\% del total) causados por serpientes (SILVA et al., 2015). Los accidentes en el trabajo que implican causas basadas en animales venenosos no se notifican de manera óptima y están subreportados. Sin embargo, hubo un aumento

$\mathrm{RC}: 67469$

Disponible: https://www.nucleodoconhecimento.com.br/salud/numero-de-accidentes 
en estos casos, teniendo en cuenta un período que abarca de 2007 a 2017 (BRASIL, 2019).

La región sureste es la campeona en el número de accidentes causados por animales venenosos, siendo las serpientes y escorpiones las principales causas de tales sucesos. Entre ellos, se ha demostrado que los escorpiones son las principales causas de este problema, ya que encuentran en las zonas urbanas una buena adaptación al medio ambiente, un factor que proporciona reproducción y consecuente proliferación de este animal en la región. Una gran proporción de accidentes de escorpión tienen consecuencias leves para el organismo humano y no requieren el uso de seroterapia. Sin embargo, en los otros casos, puede haber una amplia variación en las consecuencias de individuo a individuo, siendo la muerte un posible resultado si no hay un tratamiento adecuado. Además, puede haber secuelas de tales accidentes, con el fin de perjudicar la dinámica diaria del individuo, dificultando incluso el trabajo (LIMA et al., 2020).

Una gran proporción de casos de accidentes con animales venenosos (la mayoría de ellos) ocurren con individuos masculinos, y esta declaración es válida para la gran mayoría de los animales venenosos. En particular, entre los accidentes ófidicos, el perfil epidemiológico más común es el de los trabajadores rurales, cuya edad es entre 15 y 49 años (BRASIL, 2019). Los niños tienden a sufrir más accidentes por animales venenosos en vista de aspectos como la falta de supervisión en las actividades recreativas al aire libre, además de otras cuestiones socioculturales (NEIVA et al., 2019). El grupo de edad que comprende el mayor número de casos es de 20 a 39 años, y entre 2009 y 2013, hubo un aumento de casos en este grupo (SILVA et al., 2015a). En vista de los datos recogidos, el mayor número de personas afectadas por accidentes con animales venenosos es de etnia marrón, seguido de blancos y negros.

Entre los accidentes y muertes causadas por animales venenosos, los escorpiones son los más responsables dentro del período bajo estudio. En la región sureste (una región donde hay un mayor predominio de accidentes por animales venenosos), las

$\mathrm{RC}: 67469$

Disponible: https://www.nucleodoconhecimento.com.br/salud/numero-de-accidentes 
causas del escorpión son responsables del mayor número promedio de muertes y accidentes en esta categoría (LIMA et al., 2020). Entre los accidentes de trabajo que se producen por causas de escorpión, las partes más afectadas del cuerpo son las manos, los pies y los brazos, teniendo en cuenta el período comprendido entre 2007 y 2017 (BRASIL, 2019).

Los accidentes botrópicos corresponden al ofidismo más común del país, representando alrededor del $90 \%$ del total. El veneno tiene una serie de acciones a nivel orgánico en el individuo, pudiendo causar efectos proteolíticos, coagulantes y hemorrágicos. Además, el dolor, el edema, los hematomas y el sangrado en la región de la picadura son frecuentes. También puede haber la aparición de ampollas y infarto de grupos ganglionares (puede ocurrir necrosis de la región, pero no siempre está presente). En las mujeres embarazadas, además de las manifestaciones en cuestión, existe el riesgo de hemorragia uterina, caracterizando un caso de mayor gravedad (BRASIL, 2001).

Según los datos presentados, entre las personas que sufrieron un accidente causado por animales venenosos, la mayoría es atendida por los servicios de salud dentro de las 3 horas posteriores al accidente. El tiempo entre la picadura y el cuidado debe ser lo más pequeño posible, y de acuerdo con los datos relacionados con el estado de Rio Grande do Sul, a medida que aumenta el tiempo de atención, aumenta la posibilidad de consecuencias negativas para el individuo, como una disminución en el número de pacientes curados y un aumento de la letalidad. Aunque el mayor número de muertes ocurre entre los hombres, las hembras tienen una mayor tasa de letalidad. En el caso de pacientes de edad avanzada de 80 años o más, se observó una mayor tasa de letalidad en comparación con otros grupos de edad (DORNELES, 2009).

Los accidentes de animales venenosos, en su mayor cantidad, no representaban una clasificación basada en un estado de gravedad, es decir, la mayoría fueron clasificados como grado leve. La tasa más alta de gravedad y letalidad suele ocurrir en niños mayores e individuos (mayores de 80 años) (DORNELES, 2009). En

RC: 67469

Disponible: https://www.nucleodoconhecimento.com.br/salud/numero-de-accidentes 
relación con los accidentes de escorpión, la mayoría pueden ser tratados en una unidad de salud ubicada cerca del paciente, sin necesidad de seroterapia (LIMA et al., 2020).

Brasil es uno de los países que producen más sueros antivenom en todo el mundo (alrededor de 400.000 ampollas). A nivel nacional, el tratamiento es proporcionado por el Sistema Unificado de Salud, siendo Brasil el país cuya distribución libre de suero antiofílico se hace más integral y con mayor facilidad, ya que está disponible para cualquier herido. La cantidad producida a nivel nacional es correcta para que pueda servir al $100 \%$ de los individuos brasileños. Sin embargo, todavía hay complicaciones que deben resolverse en relación con el acceso a todas las regiones de una manera completa, con áreas indígenas y regiones remotas locales con menor disponibilidad sérica (CUNHA, 2017).

\section{CONCLUSIÓN}

Considerados como causas desatendidas de enfermedades, los accidentes causados por animales venenosos requieren una mayor atención de salud pública, y es necesario mejorar el sistema de notificación de casos y la mayor disponibilidad de información sobre el tema a la población en general. Arañas, escorpiones y serpientes son los principales causadores de estos accidentes.

El perfil epidemiológico más frecuente en accidentes odiosos es el masculino, trabajadores rurales, entre 15 y 49 años de edad. Entre los niños, los niños son los más afectados, y la falta de supervisión en las actividades diarias es una de las principales causas de este problema.

La mayoría de los accidentes de escorpión generan consecuencias menores para el organismo humano y no implican la necesidad de seroterapia. En los otros casos, sin embargo, puede haber una amplia variación en las consecuencias de paciente a paciente, y si no hay un tratamiento adecuado, el paciente puede estar yendo a la muerte.

RC: 67469

Disponible: https://www.nucleodoconhecimento.com.br/salud/numero-de-accidentes 
La mayoría de los casos suelen ser atendidos por los servicios de salud dentro de las 3 horas posteriores al accidente. El tiempo entre el accidente y la atención debe ser lo más pequeño posible $\mathrm{y}$, a medida que aumenta el tiempo de atención, la posibilidad de consecuencias negativas para el individuo aumenta proporcionalmente, con una disminución en la tasa de curación y un aumento de la letalidad.

La mayoría de los pacientes que experimentan accidentes de escorpión no requieren seroterapia, y pueden recurrir a un tratamiento adecuado en unidades de salud que se encuentran en las proximidades del lugar donde ocurrió el accidente, por lo que la locomoción es más rápida y la consiguiente tasa de mejora es mayor.

La producción de suero para el tratamiento de accidentes de odidisa en Brasil se acentúa, y la cantidad producida es capaz de cubrir el número total de casos en el país. Sin embargo, existe un problema con respecto a la distribución de este suero, ya que las zonas de población indígena y regiones de localidades remotas no tienen una disponibilidad adecuada, lo que permite consecuencias negativas para los individuos en cuestión, aumentando la posibilidad de muertes por este tipo de accidentes.

\section{REFERENCIAS}

BRASIL, Fundação Nacional de Saúde. Manual de Diagnóstico e tratamento de acidentes por animais peçonhentos, $2^{\underline{a}}$ ed., Brasília, 2001.

BRASIL, Portal da saúde. Série Histórica de Casos - Acidente Por Animais Peçonhentos. 2017. Acessado em 26 de setembro de 2017<http://portalarquivos.saude.gov.br/images/pdf/2017/abril/28/1 -

SerieHistorica_1986-2016.pdf>

RC: 67469

Disponible: https://www.nucleodoconhecimento.com.br/salud/numero-de-accidentes 
BRASIL. Secretaria de Vigilância em Saúde. Ministério da Saúde. Boletim Epidemiológico 11: Acidentes de trabalho por animais peçonhentos entre trabalhadores do campo, floresta, águas, Brasil 2007 a 2017, v. 50, 2019.

BREDT C. S, LITCHTENEKER K. Avaliação Clínica e Epidemiológica dos acidentes com animais peçonhentos atendidos no Hospital Universitário do Oeste do Paraná 2008- 2012. Rev. Med. Res., Curitiba, v.16, n.1, p. 11-17, jan./mar. 2014.

CARMO É. A, NERY A. A, JESUS C. S, CASOTTI C. A. Internaçoes hospitalares por causas externas envolvendo contato com animais em um hospital geral do interior da Bahia, 2009-2011. Epidemiol. Serv. Saude, Brasília, p.105-114, jan./mar. 2016.

CUNHA, L. E. R. Soros antiofídicos: história, evolução e futuro. Journal Health NPEPS, v. 2, supl. 1, p. 1-4, 2017.

DORNELES, A. L. Frequência de acidentes por animais peçonhentos ocorridos no Rio Grande do Sul, 2001 - 2006. Trabalho de Conclusão de curso, Especialização em Saúde Pública, Proto Alegre, RS. 2009.

LIMA E. C. F, FARIA M. D, MORAIS R. M. R. B, OLIVEIRA L. M. S. R, LIMA E. H. F, COSTA C. S. Interações entre meio ambiente, atendimentos antirrábicos e acidentes por animais peçonhentos no município de Petrolina(PE). Saúde Meio Ambient, v.6, n.1, p.54-70, jan./jun. 2017.

LIMA, C. A.; LEAL, A. L. R.; MANGUEIRA, S. A. L.; COSTA, S. M.; SANTOS, D. F. Vigilância em saúde: acidentes e óbitos provocados por animais peçonhentos na região sudeste Brasil, 2005-2015. Revista de Pesquisa: Cuidado é Fundamental Online, v. 12, p. 20-28, 2020.

LOPES A. B, OLIVEIRA A. A, DIAS F. C. F, SANTANA V. M. X, OLIVEIRA V. S, LIBERATO A. A, CALADO E. J. R, LOBO P. H. P, GUSMÃO K. E, GUEDES V. R. Perfil Epidemiológico dos Acidentes por Animais Peçonhentos na Região Norte

RC: 67469

Disponible: https://www.nucleodoconhecimento.com.br/salud/numero-de-accidentes 
Entre os Anos entre 2012 e 2015: Uma Revisão. Revista de Patologia do Tocantins, v.4, n.2, p.36-40, 2017.

NEIVA, C.A.C et al. Caracterização epidemiológica das intoxicações exógenas por substâncias nocivas e acidentes por animais peçonhentos em crianças no Estado do Amapá. Revista Científica Multidisciplinar Núcleo do Conhecimento, v. 01, p. 41-66, 2019. https://www.nucleodoconhecimento.com.br/saude/caracterizacaoepidemiologica

SALOMÃO, M. G.; LUNA, K. P. O.; MACHADO, C. Epidemiologia dos acidentes por animais peçonhentos e a distribuição de soros: estado de arte e a situação mundial. Rev. Salud Pública, v. 20, n. 4, p. 523-529, 2018.

SANTANA V. T. P, SUCHARA E. A. Epidemiologia dos acidentes com animais peçonhentos registrados em Nova Xavantina - MT. Revista de Epidemiologia e Controle de Infecção, v.5, n.3, p.141-146, 2015.

SILVA C. T., PARDAL P. P. Atividades Lúdicas Na Prevenção De Acidentes Por Animais Peçonhentos Em Estudantes Da Rede Pública Da Região Metropolitana De Belém. Revista Universo \& Extensão, v.3, n.3, 2015.

SILVA, A. M.; BERNARDE, P. S.; ABREU, L. C. Acidentes com animais peçonhentos no Brasil por sexo e idade. Journal of Human Growth and Development, v. 25, n. 1, p. 54-62, 2015a.

SILVA J. H, GIANSANTE S, SILVA R. C. R, SILVA G. B, SILVA L. B, PINHEIRO L. C. B. Perfil Epidemiológico dos Acidentes com Animais Peçonhentos em Tangará da Serra-Mt, Brasil (2007-2016). Journal Health NPEPS, p.5-15, 2017.

SOUZA C. M. V, MACHADO C. Animais peçonhentos de importância médica no município do rio de janeiro. Journal Health NPEPS, p.16-39 2017.

Artículo: Diciembre de 2020.

$\mathrm{RC}: 67469$

Disponible: https://www.nucleodoconhecimento.com.br/salud/numero-de-accidentes 
Aprobado: Diciembre de 2020.

RC: 67469

Disponible: https://www.nucleodoconhecimento.com.br/salud/numero-de-accidentes 